\title{
Wax-Crystal Modification for Fuel Oils by Self-Aggregating Partially Crystallizable Hydrocarbon Block Copolymers
}

\author{
Walter Leube, Michael Monkenbusch, Dieter Schneiders, and Dieter Richter \\ Institut für Festkorperforschung Forschungszentrum, J ülich GmbH, J ülich, Germany D-52425 \\ Douglas Adamson ${ }^{\dagger}$ and Lewis Fetters* \\ ExxonMobil Research and Engineering Co., Strategic Corporate Research Laboratories, \\ 1545 Rt. 22 East, P.O. Box 998, Annandale, New J ersey 08801-0998 \\ Panagiotis Dounis and Ralph Lovegrove \\ Infineum, Ltd., Milton Hill, Abingdon, England OX13 6BB
}

Received J uly 15, 1999. Revised Manuscript Received J anuary 4, 2000

\begin{abstract}
The qual ity of life partially depends on the ability to maintain the transportation of goods and people in an efficient and reliable fashion, even under difficult climatic conditions. A well-known problem at low temperatures is the filter blockage of diesel fuel. Fuel oils contain alkanes that precipitate at low temperature as large crystals or spherulites of wax in such a way as to form gels. Thereupon the fuel loses its ability to flow and the transportation system falters or is stopped. The lowest temperature at which the fuel will still flow is known as the pour point. As the temperature approaches that of the pour point, difficulties emerge in transporting the fuel through lines and pump. Furthermore, wax crystals can plug screens and filters at temperatures above the pour point; the so-called cold filter plugging point. To combat this behavior various additives have been devel oped to depress the pour point or to decrease the size and alter the shape of the wax crystals; e.g., smaller sized crystals are less likely to d og either screens or filters. This paper reports on the use of a crystalline-amorphous diblock copolymer that performs, in its selfassembled state, as an efficient nucleator for the wax in middle distillate fuels.
\end{abstract}

\section{Introduction}

Recently, we have reported on the structure of polymer aggregates formed from polyethylene-poly(ethylene-propylene) diblock copolymers (PE-PEP) in decane. ${ }^{1,2}$ Such diblocks self-assemble to form hairy platel et structures where the perpendicular dimensions are controlled by molecular weight and composition ratios. Figure 1 displays, in the form of a cartoon, the structural information that was obtained by small-angle neutron scattering (SANS) experiments ${ }^{1}$ for sample molecular weights of the order of $10^{4}$. The diblock copolymers form core PE plates, with a thickness range of 20-100 $\AA$, surrounded on both sides by the PEP polymer brush where the hairs reach out about $200 \AA$.

The diblock copolymers studied in this work were derived from poly(butadiene-isoprene) diblocks prepared by anionic polymerization and subsequent hydrogenation. The spontaneous occurrence of 1,2-addition in

* Corresponding author.

+ Present address: Department of Materials Science, Princeton University, Princeton, NJ 08544.

(1) Richter, D.; Schneiders, D.; Monkenbusch, M.; Willner, L.; Fetters, L. J .; Huang, J. S.; Lin, M.; Mortenson, K.; Farago, B. Macromolecules 1997, 30, 1053-1068.

(2) Montkenbusch, M.; Schneiders, D.; Richter, D.; Farago, B.; Fetters, L.; Huang, J . II Nuovo Cimento 1994, 16, 747-755. the butadiene segment leads to ethyl side branches in the polyethylene chains (about 2 per 100 main-chain carbon atoms). These side branches lead to defect structures in the polyethylene crystals and result in a relatively rough surface (see lower part of Figure 1 ). The right half of Figure 1 presents the superstructure; namely, the stacking of these platelets to higher order aggregates. ${ }^{1}$ Typical stacks contain about 10 to 12 platelets. These diblock copolymers can serve as wax nucleators in n-decane; ${ }^{3}$ a topic which has been the subject of a theoretical treatment by Buzza and McLiesh. ${ }^{4}$ Empirically, these diblock systems have proven to exhibit nucleator activity in various diesel fuels.

In this paper the thermodynamics of the platelet formation are discussed and relations derived. The platelet surface area is predicted to depend on the diblock composition and segment molecular weights. We present SANS experiments on such diblock copolymer aggregates in the presence of wax. Using contrast variation we directly visual ize the nucleation properties of these materials.

(3) Schneiders, D. Ph.D. Thesis. University of Aachen, Aachen, Germany, September 25, 1996.

(4) Buzza, D. M. A.; McLiesh, T. C. B. J . Phys. II France 1997, 7, 1379-1392. 


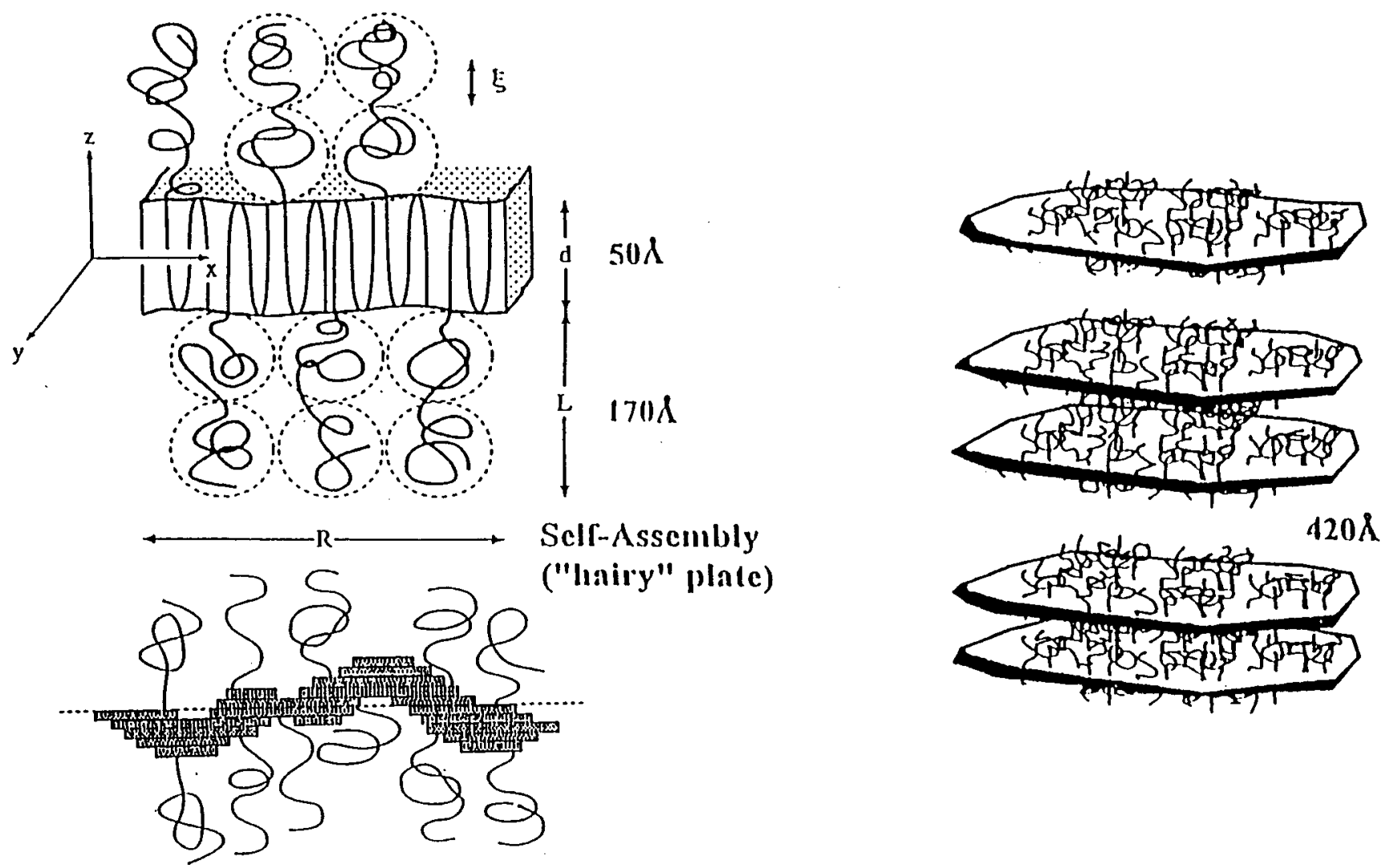

Figure 1. Generic structure of the PE-PEP aggregates. The crystallization of the PE forms extended (R) platelets, the PEP hairs form brushes on both sides. The length $d$ and $L$ vary with molecular weights. The lower part indicates that the crystallization core exhibits some amount of surface roughness.

\section{Theoretical}

Aggregation and Platelet Formation. The thermodynamics of platelet formation are governed by the gain of crystallization enthalpy opposed by the energetic penalty of the entropy increase due to chain stretching in the brush (see Figure 1) and the enthalpy of chain fol ding at the core surface. In addition, as will be seen, the defect energies of the ethylene side chains (which are present as a consequence of the anionic polymerization process) play an important role. We discuss first polymer brush behavior in terms of the model of Alexander ${ }^{5}$ and de Gennes. ${ }^{6}$ They treat the polymer brush in analogy with semidilute polymer solutions. In this approach each hair can be considered as a string of blobs reaching out from the surface. The blob size describes the screening of the excluded volume interaction due to neighboring chains. Its size $\xi$ relates to the surface density of blobs $\Omega$.

$$
\xi \approx \Omega^{-0.5}
$$

The hair surface density is directly related to the core thickness $d$ and the chain length $N_{P E}$ of the polyethylene subchain:

$$
\Omega=\frac{0.5 d}{\mathrm{v}_{0}^{\mathrm{PE}} \mathrm{N}_{\mathrm{PE}}}
$$

where $\mathrm{v}_{0}{ }^{\mathrm{PE}}$ denotes the volume of a monomer in the PE

(5) Alexander, S. J . Phys. France 1977, 38, 983-990.

(6) de Gennes, P.-G. Macromol ecules 1980, 13, 1069-1075. sub-chain. Inside a blob the chains are swollen and we have:

$$
\xi \approx \lg ^{v}
$$

where $I$ is the length of a monomer, $g$ the number of monomers in the blob, and $v \cong 0.6$ is the Flory exponent. With the condition that the density inside one bl ob has to be equal to the averaged density this consideration immediately leads to an expression for the brush length $\mathrm{L}_{\mathrm{p}}$ :

$$
L_{p}=\left.N_{P E P}\left[0.5 d / N_{0}{ }^{P E} N_{P E}\right]^{1 / 3}\right|^{5 / 3}
$$

Hence, $N_{\text {PEP }}$ represents the number of monomers in the poly(ethylene-propylene) segment. This derivation of $L_{p}$ requires the assumption of a rectangular brush profile whereas SANS has shown that the actual profile is closer to parabolic. This does not, however, alter the scaling result.

We now turn to the free energy of the aggregate. Two contributions must be considered. The chain stretching within the brush leads to a loss of conformational entropy that can be estimated by the number of blobs $\left(n_{b}\right)$ per chain. Each blob contributes $k T$ to the free energy. Since $n_{b}=L_{p} / \xi$ with eqs 1,2 , and 4 we obtain

$$
\mathrm{F}_{\text {brush }}=\mathrm{k}_{\mathrm{b}} \mathrm{TN}_{\mathrm{PEP}} \mathrm{P}^{5 / 3} \Omega^{5 / 6}
$$

The enthal pic part of the free energy contains two contributions. The first results from the chain folding and is simply given by the number of chain folds $n_{f}$ times the fold energy $E_{f}=170 \mathrm{meV}$ : 


$$
\mathrm{F}_{\text {core }}=\mathrm{E}_{\mathrm{f}} \mathrm{n}_{\mathrm{f}}=\frac{2 \mathrm{E}_{\mathrm{f}} \mid \mathrm{N}_{\mathrm{PE}}}{\mathrm{d}}=\frac{\mathrm{E}_{\mathrm{f}}}{\eta_{0}{ }^{\mathrm{PE}} \Omega} \mid
$$

As has been recently shown, ${ }^{1}$ there exists a further important contribution to the core free energy resulting from the ethyl side-branches. These branches, when incorporated into the PE crystal, will cause high defect energies. This topic has been investigated by Gaucherie and Seguela, ${ }^{7}$ who measured the change in melting enthalpy $\mathrm{H}_{\mathrm{f}}$ of PE crystals as a function of total ethyl group concentration. For a material equivalent to ours they arrive at $\mathrm{H}_{\mathrm{f}}=75 \mathrm{~J} \mathrm{gm}^{-1}$ which yields a defect energy of $E_{\text {def }} \cong 600 \mathrm{meV}$. They also found a strong tendency of the system to expel the ethyl branches into the amorphous phase. In other words, the ethyl branch frequency leads to more frequent chain folding in order to avoid ethyl group incorporation in the bulk. With a low number of ethyl branches in our PE chains the Poisson distribution of side groups is very close to an equal distribution yielding a rather uniform probability of $p=0.017$ of finding an ethyl unit at any backbone carbon. An increase in the PE surface area serves to enhance the probability of locating an ethyl branch in the vicinity of the brush-platelet surface. In the neighborhood of the surface it is an obvious assumption that the energy cost of incorporating a branch into the crystal is markedly diminished, i.e., in the region of a fold these energy costs are minimized. In this spirit an expression can be formulated for the defect contribution to the aggregate free energy:

$$
F_{\text {def }}=\left[F^{0}\right]_{\text {def }}-n_{f} n_{s} p E_{\text {def }}=[F]_{\text {def }}-n_{s} p E_{\text {def }} \frac{1}{\Omega v_{0}^{P E}}
$$

where $\left[\mathrm{F}^{0}\right]_{\text {def }}$ is a defect energy for the incorporation of all the ethyl branches into a large PE crystal and $n_{s}$ denotes the number of methylene units in the neighborhood of the fold at which the energy costs for ethyl group incorporation are strongly reduced. The product $\left(n_{\mathrm{f}} n_{\mathrm{s}} p\right)$ represents the number of ethyl units that do not contribute to the defect energy. We note that $F_{\text {def }}$ scales as $F_{\text {core }}$ with $\Omega^{1-}$ (second part of eqn [7]). Thus, the scaling properties of the total free energy are not affected by the addition of this defect term. Finally, the total free energy may be written as:

$$
\mathrm{F}=\frac{\mathrm{I}}{\mathrm{v}_{0}^{\mathrm{PE}}}\left(\mathrm{E}_{\mathrm{f}}-\mathrm{n}_{\mathrm{s}} \mathrm{pE} \mathrm{E}_{\text {def }}\right) \Omega^{-1}+\left.\mathrm{kTN}_{\mathrm{PEP}}\right|^{5 / 3} \Omega^{5 / 6}
$$

Minimization of the total free energy with respect to $\Omega$ leads to additional scaling relations:

$$
\begin{gathered}
d=C_{1} N_{P E} N_{P E P}^{-6 / 11} \\
L_{p}=C_{2} N_{P E P}^{9 / 11}
\end{gathered}
$$

The prefactors $C_{1}$ and $C_{2}$ can be determined from experiment.

In the introduction it was inferred that wax crystal nucleation occurs at the polyethylene surface of the platelets. Therefore, it is of importance to evaluate the

(7) Gaucherie, V.; Seguela, R. Polymer 1994, 35, 2049-2055.
Table 1. Representative Molecular Characteristics of PE-PE P Crystalline-Amorphous Di-Block Copolymers

\begin{tabular}{lcr}
\multicolumn{1}{c}{ polymer } & \multicolumn{1}{c}{$6 / 10$} & \multicolumn{1}{c}{$1.5 / 5$} \\
\hline$M_{\text {PE }}\left(\mathrm{g} \mathrm{mol}^{-1}\right)$ & $6000( \pm 5 \%)$ & $1500( \pm 5 \%)$ \\
$M_{\text {PEP }}\left(\mathrm{g} \mathrm{mol}^{-1}\right)$ & $10000( \pm 5 \%)$ & $5000( \pm 5 \%)$ \\
$\rho_{\text {PE }}\left(\mathrm{g} \mathrm{mol}^{-3}\right)$ & $0.93( \pm 0.02)$ & $0.93( \pm 0.02)$ \\
$\rho_{\text {PEP }}\left(\mathrm{g} \mathrm{mol}^{-3}\right)$ & $0.85( \pm 0.01)$ & $0.85( \pm 0.01)$ \\
$\nu_{\text {PE }}$ & $0.38( \pm 0.02)$ & $0.19( \pm 0.02)$ \\
$m_{S}$ & $0.82( \pm 0.06)$ & $1.67( \pm 0.12)$ \\
$\xi_{\text {PE }} / 10^{10}\left(\mathrm{~cm}^{-2}\right)$ & $-0.33( \pm 0.01)$ & $-0.33( \pm 0.01)$ \\
$\xi_{\text {PEP }} / 10^{10}\left(\mathrm{~cm}^{-2}\right)$ & $5.06( \pm 0.77)$ & $5.06( \pm 0.77)$ \\
$A P S A\left(\mathrm{~m}^{2} \mathrm{~g}^{-1}\right)$ & $173^{\mathrm{a}} / 36^{\mathrm{b}}$ & $251^{\mathrm{a}} / 33^{\mathrm{b}}$
\end{tabular}

a Ethyl branches present. b Zero ethyl branch content.

available platelet surface area (APSA) per gram of block copolymer in the fuel. We start with a calculation of the surface area per PE chain. Considering the molecular weight of the polyethylene chains $M_{P E}$ and the polyethylene density $\rho_{\mathrm{PE}}$ the surface per chain SA becomes:

$$
\mathrm{SA}=\frac{2 \mathrm{M}_{\mathrm{PE}}}{\mathrm{d} \rho_{\mathrm{PE}} \mathrm{N}_{\mathrm{a}}}
$$

where $d$ should be taken from eq 9 with the prefactor $\mathrm{C}_{1}$ obtained from SANS measurements and $\mathrm{N}_{\mathrm{a}}$ is the Avogadro number. From this surface we have to subtract the surface occupied by one hair:

$$
\mathrm{S}_{\text {hair }}=\delta \frac{\vartheta^{\mathrm{PE}}}{\mathrm{l}}=\delta \frac{\mathrm{m}_{\mathrm{o}}^{\mathrm{PE}}}{\rho_{\mathrm{PE}} \mathrm{IN}}
$$

Hence, $\vartheta^{\mathrm{PE}}$ is the volume of a polyethylene monomer and $I$ its length. The factor $d$ all ows an effectively larger surface area than is blocked by the PEP hairs. Subtracting eq 12 from eq 11 yields the available surface area per chain. If we now multiply by the number of chains per gram we arrive at the required quantity the available platelet surface area:

$$
\begin{aligned}
& \text { APSA }= \\
& \left(\frac{1}{\rho_{P E} N_{a}}\right)\left(\frac{m_{0}{ }^{P E P}}{m_{0}{ }^{P E} N_{P E}+m_{0}{ }^{P E P} N_{P E P}}\right)\left(\frac{2 N_{P E P}{ }^{6 / 11}}{C_{1}}-\frac{\delta}{l}\right)
\end{aligned}
$$

There $m_{0}{ }^{P E}$ and $m_{0}{ }^{P E P}$ denote the molecular weights of the respective monomers. At this point we will consider monomers being a section of the backbone with 4 methylene units (or monomers) having 4 carbons each in the backbone. This methodology relates the polyolefinic structure to the parent polydiene diblock. The value of eq 13 is that it predicts that platlet surface area is jointly dependent upon diblock composition and segment molecular weights. Two examples are given in Table 1. It is seen that the linear polyethylene segments yield noticably lower APSA values than the corresponding segments containing ethyl branches. ${ }^{1}$

Small-Angle Neutron Scattering (SANS). SANS uses wavelengths $\lambda$ between ca. 5 and $15 \AA$ over an angular range of about $0.1^{\circ}$ to $20^{\circ}$. Therefore it probes structural dimensions between 15 and several thousand $\AA$. Since in the smallest of these spatially resolvable volumes there are many atoms, the system to be investigated may be described in terms of scattering length densities $\xi_{\mathrm{i}}$ ignoring individual atom positions. 
The advantage of neutrons over X-rays is the ability to vary the scattering length densities of different constituents of a hydrocarbon sample over a broad range by $H / D$-substitution. Since the molecules affected by H/D-exchange are chemically the same, the physical chemistry of the samples is-if at all-only marginally modified. The visibility of an object, in solution for example, depends on the difference of the solvent/solute scattering length densities as shown:

$$
\Delta \zeta_{\mathrm{m}}=\frac{\sum_{\mathrm{i}=1}^{\text {atoms in } \mathrm{M}} \mathrm{b}_{\mathrm{i}}^{\mathrm{M}}}{\mathrm{v}_{\mathrm{M}}}-\frac{\sum_{\mathrm{j}=1}^{\text {atoms in } \mathrm{S}} \mathrm{b}_{\mathrm{j}}^{\mathrm{S}}}{\mathrm{v}_{\mathrm{S}}}
$$

where $b_{i}{ }^{M}$ denotes the scattering lengths of the (different) atoms in $\mathrm{M}$ and $\mathrm{b}_{j}^{\mathrm{S}}$ those of the atoms in $\mathrm{S}$. $\mathrm{V}_{\mathrm{m}}$ and $V_{s}$ are the effective volumes occupied by the objects composed of the atoms in the respective sums; e.g., M may denote a monomer segment of a polymer and $\mathrm{S}$ a solvent molecule. Since $b_{H}=-3.57 \times 10^{-13} \mathrm{~cm} ; b_{D}=$ $6.57 \times 10^{-13} \mathrm{~cm} ; b_{c}=-6.65 \times 10^{-13} \mathrm{~cm}$. H/D replacement allows for huge variations of $\Delta \xi$ for hydrocarbons.

By using a mixture of suitable amounts of deuterated and protonated solvent it is possible to achieve zero contrast, $\Delta \xi_{M}=0$, for one component, $M$, of the system. This technique of contrast matching and variation is essential for the investigation of complicated multicomponent systems as $\mathrm{A}-\mathrm{B}$ - block copolymer aggregates and the associated wax molecules.

The contribution of scattering intensity from some object described by its scattering length density distribution $\Delta \xi(\vec{r})$ is:

$$
\begin{gathered}
\mathrm{I}(\theta)=\left(\mathrm{TI}_{\mathrm{o}}\right) \frac{\mathrm{d} \sigma}{\mathrm{d} \Omega} \text { with } \Delta \Omega \text { and } \mathrm{Q}=\frac{4 \pi}{\lambda} \sin (\theta / 2) \\
\frac{\mathrm{d} \sigma}{\mathrm{d} \Omega}=\left|\int \Delta \xi(\overrightarrow{\mathrm{r}}) \mathrm{e}^{\mathrm{i} \overrightarrow{\mathrm{Q}} \vec{r}} \mathrm{~d}^{3} \overrightarrow{\mathrm{r}}\right|^{2}
\end{gathered}
$$

where I $(\theta)$ is the intensity scattered into one detector el ement, $\Delta \Omega$ the solid angle covered by that element, $\theta$ the scattering angle, and $I_{0}$ the incoming neutron flux and $\mathrm{T}$ a sample transmission factor. The structural information is contained in $\mathrm{d} \sigma / \mathrm{d} \Omega$. It is the absolute square of the Fourier transform of $\Delta \xi(\vec{r})$. The squaring and the orientational averaging necessary for anisotropic objects in an isotropic solution prevents direct calculation of $\Delta \xi(\vec{r})$ from $I(\theta)$ by a simple inverse transform. For exact structural details one has to rely on model fitting (see below). To gain initial information on the structure some integral properties may be deduced from the forward scattering I (0) and the power $\gamma$ provided by $\mathrm{d} \sigma / \mathrm{d} \Omega \sim \mathrm{Q}^{\gamma}$ in varying Q-regimes.

With neutrons it is comparatively easy to obtain scattering data on an absolute scale normalized to the sample volume. For solutions $\mathrm{V}_{\text {sample }}$ is taken as the volume of dissolved polymer:

$$
\frac{\mathrm{d} \Sigma}{\mathrm{d} \Omega}=\mathrm{V}_{\text {sample }}{ }^{-1} \frac{\mathrm{d} \sigma}{\mathrm{d} \Omega}
$$

The expression $\mathrm{d} \sigma_{\mathrm{d}} / \mathrm{d} \Omega=(\mathrm{Q} \rightarrow 0)=\left|\int \Delta \xi(\vec{r}) \mathrm{d}^{3} \mathrm{r}\right|$ equals $\Delta \xi^{2} \times \mathrm{V}_{0}^{2}$; where $\mathrm{V}_{0}$ is the volume of the object, i.e., an aggregate, here assumed to be homogeneously decorated with the constant contrast factor $\Delta \xi$. If $\mathrm{N}$ of these objects are located at random positions in a solution-since all interference average out to zero-their scattering intensities add up to $\mathrm{N}(\mathrm{Q})\left(\mathrm{d} \sigma_{\mathrm{o}} / \mathrm{d} \Omega\right)$. A polymer volume $\mathrm{V}$ in solution that splits into $\mathrm{N}$ aggregates yields $\mathrm{V}_{0}=$ $\mathrm{V} / \mathrm{N}$ and

$$
\begin{aligned}
\frac{\mathrm{d} \Sigma\left(\mathrm{Q}^{\circ}\right)}{\mathrm{d} \Omega}=\frac{1}{\mathrm{~V}} \frac{\mathrm{d} \sigma}{\mathrm{Vd} \Omega}(\mathrm{Q} \rightarrow 0)=\frac{\mathrm{N}_{\mathrm{ag}}}{\mathrm{V}} \Delta \xi^{2}\left(\frac{\mathrm{V}}{\mathrm{N}_{\mathrm{ag}}}\right)^{2}= \\
\Delta \xi^{2} \frac{\mathrm{V}}{\mathrm{N}_{\mathrm{ag}}}=\Delta \xi^{2} \frac{\mathrm{N}_{\mathrm{ag}} \mathrm{M}}{\rho \mathrm{N}_{\mathrm{L}}}
\end{aligned}
$$

where $\mathrm{N}_{\mathrm{ag}}$ is the aggregation number, $\mathrm{M}$ the polymer molecular weight, $\rho$ the bulk (effective) polymer mass density, and $\mathrm{N}_{\mathrm{a}}$ the Avogadro number. The aggregation number is immediately accessible by the extrapolation of the scattering intensity toward zero angle. A typical polymer solution with $\mathrm{M}_{\mathrm{w}}$ of $10^{4} \mathrm{~g} / \mathrm{mol}$ with a contrast factor $\Delta \zeta=5 \times 10^{-2} \mathrm{~cm}$ yields $\mathrm{d} \Sigma / \mathrm{d} \Omega(\mathrm{Q} \rightarrow 0) \approx 50 \mathrm{~cm}^{-1}$.

Scattering from a Platelet. The neutron scattering cross section for hairy platel ets has been presented. ${ }^{1}$ Herein we repeat the main reasoning, explain the scattering function of the structural model, and report the main results. It is easy to write down the scattering from a homogeneous rectangular "tile" with dimension $L_{x}, L_{y}$, and $d:$

$$
\begin{aligned}
\frac{\mathrm{d} \sigma}{\mathrm{d} \Omega}(\overrightarrow{\mathrm{Q}})=\Delta \xi^{2}\left|\int_{0}^{\mathrm{d}} \int_{0}^{\mathrm{L}_{y}} \int_{0}^{\mathrm{L}_{\mathrm{x}}} \mathrm{e}^{-\overrightarrow{\mathrm{Q}} \cdot \overrightarrow{\mathrm{r}}} \mathrm{d}^{3} \overrightarrow{\mathrm{r}}\right|^{2}= \\
\Delta \xi^{2} \mathrm{R}\left(\mathrm{d}, \mathrm{Q}_{\mathrm{z}}\right) \mathrm{R}\left(\mathrm{L}_{\mathrm{y}}, \mathrm{Q}_{\mathrm{y}}\right) \mathrm{R}\left(\mathrm{L}_{\mathrm{x}}, \mathrm{Q}_{\mathrm{x}}\right)
\end{aligned}
$$

with the function $\mathrm{R}$ :

$$
\begin{aligned}
\mathrm{R}(\mathrm{a}, \mathrm{q})=4\{\sin (\mathrm{a} / 2 \times \mathrm{q}) / \mathrm{q}\}^{2} \approx \\
\mathrm{a}^{2}(2 \pi / 6)^{1 / 3} \mathrm{e}^{-\left(\mathrm{a}^{2} \mathrm{q}^{2} / 12\right) \times(3 / \pi) 1 / 3}
\end{aligned}
$$

This function has narrow distributions in reciprocal space if the real space extension is large. For the large platelet dimensions $L_{x}$ and $L_{y}$ the Gaussian approximation for $\mathrm{R}$ applies (see Figure 2). This is a dual consequence of the fact that platelet shape is neither uniform nor rectangular so that the oscillations of (sin$[\mathrm{QL} / 2] / \mathrm{Q})^{2}$ are averaged out and that the relevant intensity seen in the isotropically averaged scattering stems from the region $|\mathrm{LQ}|<\pi$.

As seen in Figure 2 the reciprocal space intensity has the shape of a rod along the $\mathrm{Q}_{z}$ axis. Any modulation of the density profile of the platelet along $z$ yields a form factor:

$$
P\left(Q_{z}\right)=\left|F\left(Q_{z}\right)\right|^{2}=\left|\int \rho(z) e^{-i Z q_{z}} d q_{z}\right|^{2}
$$

that modulates the intensity of the rod along its axis for a finite width $F\left(Q_{z}\right)=R\left(d, Q_{z}\right)$. The thickness of the rod is determined by $\mathrm{L}^{-1}$ the lateral extension of the plate:

$$
F\left(Q_{z}\right) \sim A^{2}\left(\frac{2 \pi}{6}\right)^{2 / 3} \exp \left\{-\frac{L^{2}\left(Q_{x}{ }^{2}+Q_{y}{ }^{2}\right)}{12}\left(\frac{3}{\pi}\right)^{1 / 3}\right\}
$$

where $A$ is the platelet area.

Angular Averaging. For an isotropic ensemble of platelets the detection of scattering takes place on a sphere of radius Q in the platelet-associated coordinate system shown in Figure 2. The detection element is 


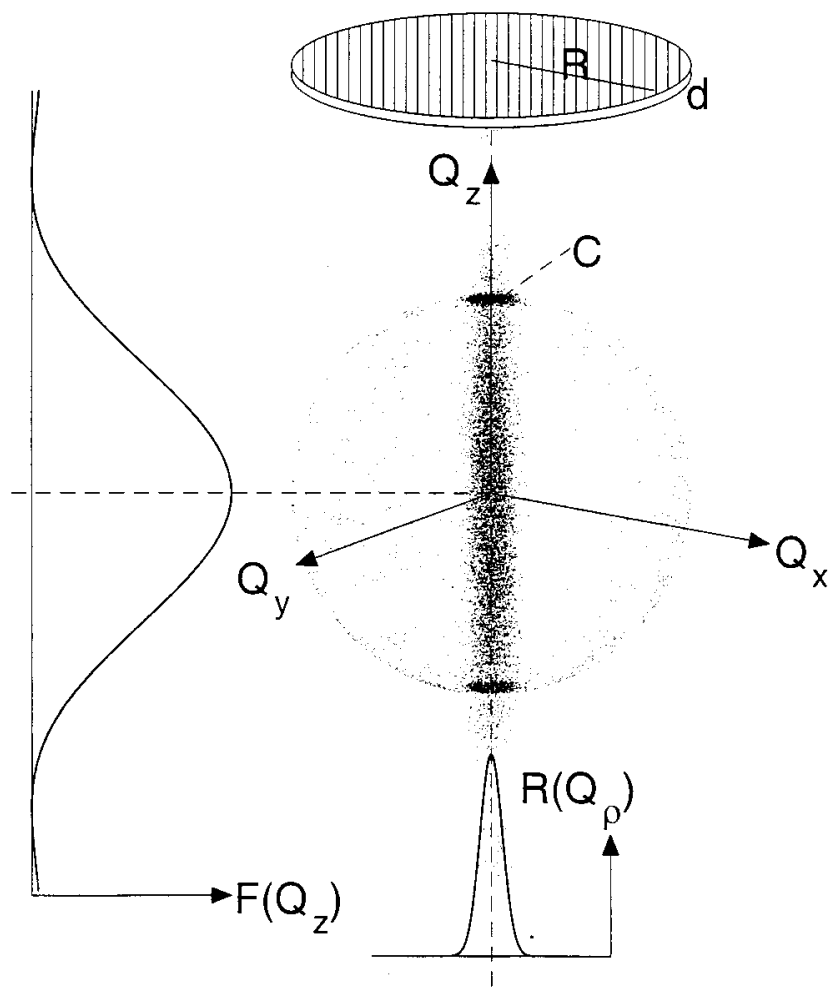

Figure 2. Reciprocal space intensity from a platel et of finite thickness and large but not infinite lateral extension. The intensity is located on a rod along $\mathrm{Q}_{z}$ which is modulated by the form factor $\left|\mathrm{F}\left(\mathrm{Q}_{\mathrm{z}}\right)^{2}\right|$ of the density profile perpendicular to the surface. The thickness of the rod is indicated by the Gaussian approximation R(Q). Its width is inversely proportional to the lateral extension. The sphere represents the locations at which the reciprocal space intensity matches the instrumental acceptance if set to a Q-value equal to the radius of the sphere. Each dot on the sphere may be considered as one orientation present in a random ensemble of platelets, i.e., an isotropic solution. The overlap of the sphere and the rod, indicated by $\mathrm{C}$, yiel ds the measured intensity. Due to increase of the sphere surface $\sim Q^{2}$ the intensity combination due to $C$ is $\sim Q^{-2}$ as long as $C$ is only a small spot on the sphere as in this figure.

"diluted" on this surface, e.g., by a factor $\left(4 \pi Q^{2}\right)^{-1}$. The intensity contributions stem only from regions where the A-sphere intersects the rod. For the outer part this immediately yields the $\mathrm{Q}^{-2}$ behavior of the scattering from thin platelets, i.e., $\mathrm{R}(\mathrm{d}, \mathrm{Q}) \approx \mathrm{R}(\mathrm{d}, \mathrm{Q}=0)$. The divergence implied by the above power law, however, is avoided by the total immersion of a very small Q-sphere in the rod. There, just the finite inner intensity contribution of the rod is accumulated completely for all orientations. This yields a scattering intensity proportional to the size of the aggregate, see eq 17. This situation is depicted in Figure 3.

This may also be seen analytically if disklike platelets with radial symmetry are assumed; then $R\left(L_{x}, Q_{x}\right)$ and $\mathrm{R}\left(\mathrm{L}_{\mathrm{y}}, \mathrm{Q}_{\mathrm{y}}\right)$ are replaced by

$$
\left|2 \pi R^{2}{ }_{1}\left(Q_{p} R\right) /\left(Q_{p} R\right)\right|^{2} \cong\left(\pi R^{2}\right)^{2} e^{-0.5 Q_{p}^{2} R^{2}}
$$

The radial averaging reads as:

$$
\begin{aligned}
&\left\langle\mathrm{I}_{\mathrm{rad}}(2)\right\rangle=\int_{6}^{\pi / 2}\left(\pi \mathrm{R}^{2}\right)^{2} \mathrm{e}^{-0.5(\mathrm{QR} \sin \vartheta)^{2}} \sin \vartheta \mathrm{d} \vartheta= \\
&\left(\pi \mathrm{R}^{2}\right)^{2} \frac{\mathrm{D}(\mathrm{QR} / 2)}{\mathrm{QR} / 2}
\end{aligned}
$$

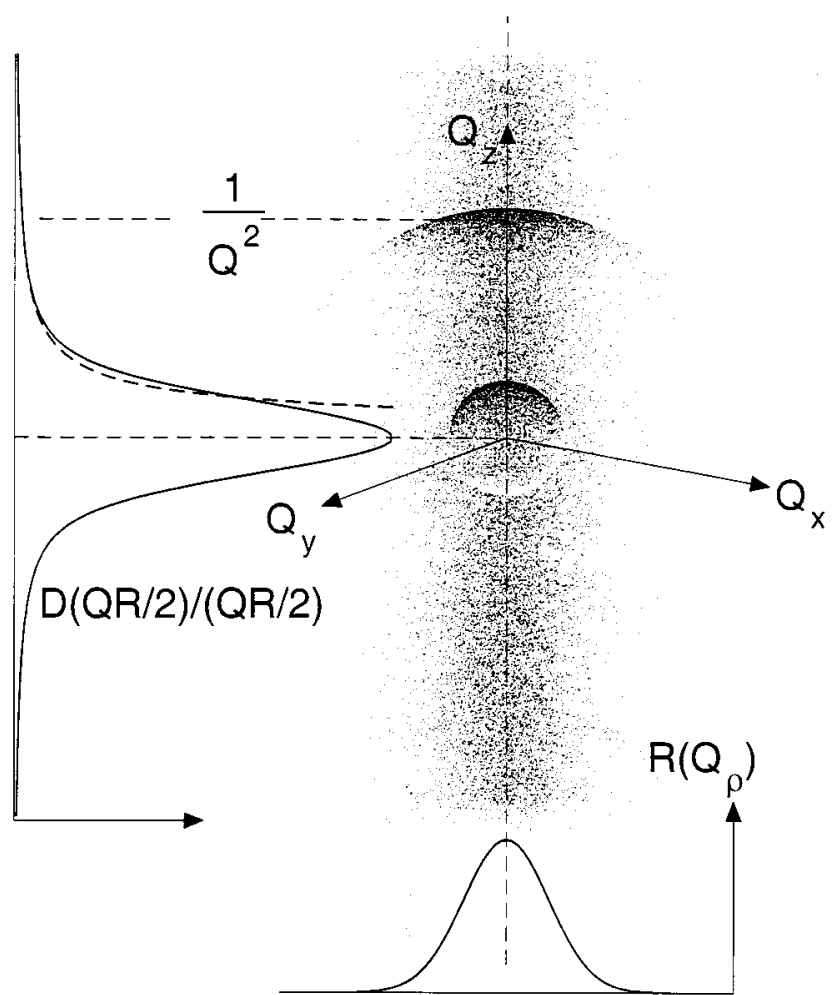

Figure 3. Enlarged view on the intensity rod in reciprocal space as shown in Figure 2. Here the overlap function $D(Q R /$ $2) /(Q R / 2)$, solid line on the left (see text) and its limiting $Q^{-2}$ behavior are plotted. The modulating form factor $\left|F\left(Q_{z}\right)^{2}\right|$ is virtually constant in this regime. The situation for a larger $\mathrm{Q}$-sphere in the $\mathrm{Q}^{-2}$ regime and that for a largely immersed sphere is depicted and may be associated with the corresponding parts of the $D(Q R / 2) /(Q R / 2)$ function.

where $\mathrm{R}$ is the platelet radius and $\mathrm{D}$ denotes the Dawson function which displays the following asymptotic behavior:

$$
\lim _{\mu \rightarrow \infty}\left(\frac{\mathrm{D}(\mu)}{\mu}\right) \rightarrow \frac{1}{2 \mu^{2}} \text { and } \lim _{\mu \rightarrow \infty}\left(\frac{\mathrm{D}(\mu)}{\mu}\right) \rightarrow 1
$$

Thus, the assumption of a finite disk removes this divergencey. Necessary modifications for elongated platelets are discussed in ref 1 . The other well-known behavior $\mathrm{I} \sim \mathrm{Q}^{-4}$ for Porod scattering is immediately derived if for thicker platelets $Q F\left(Q_{z}\right)=R\left(d, Q_{z}\right) \sim$ $\mathrm{Q}^{-2} \mathrm{z}$ contributes only if $\overrightarrow{\mathrm{Q}} \approx \overrightarrow{\mathrm{Q}}_{\mathrm{z}}$ with a factor $\mathrm{D}(\mathrm{QR} / 2) /$ $(\mathrm{QR} / 2) \sim \mathrm{Q}^{-2}$.

Our hairy platelets are not infinitely thin but have a lateral profile. This lateral profile gives rise to a form factor which modulates the profile of the Bragg rods. The form factor relates directly to the volume fractions $c_{b}(z)$ of the brush and $c_{c}(z)$ of the core, where $z$ is a coordinate perpendicular to the platelet surface. The form factor $\mathrm{P}(\mathrm{Q})$ then is given by

$$
\begin{aligned}
\mathrm{P}(\mathrm{Q})= & \left|\int_{-\infty}^{+\infty}\left[\Delta \xi_{b} c_{b}(z)\right] e^{\mathrm{i} \mathrm{Q}_{z}} \mathrm{dz}\right|^{2}= \\
& \Delta \zeta_{\mathrm{b}}{ }^{2} \mathrm{P}_{\mathrm{bb}}(\mathrm{Q})+2 \Delta \zeta_{\mathrm{b}} \Delta \zeta_{c} \operatorname{Re}\left\{\mathrm{P}_{\mathrm{bc}}(\mathrm{Q})\right\}+\mathrm{P}_{\mathrm{cc}} \mathrm{Q}
\end{aligned}
$$

$\Delta \xi_{\mathrm{b}}$ and $\Delta \xi_{\mathrm{c}}$ are the scattering length contrasts of the core and the brush polymer:

$$
\Delta \xi_{\mathrm{b}}=\frac{\Sigma \mathrm{b}_{\mathrm{b}}}{v_{\mathrm{b}}}-\frac{\Sigma \mathrm{b}_{\mathrm{s}}}{v_{\mathrm{s}}}
$$




$$
\Delta \xi_{\mathrm{c}}=\frac{\Sigma \mathrm{b}_{\mathrm{c}}}{v_{\mathrm{c}}}-\frac{\Sigma \mathrm{b}_{\mathrm{s}}}{v_{\mathrm{s}}}
$$

where $b_{b}, b_{c}$, and $b_{s}$ are the scattering lengths of the atoms forming the monomers of the brush, the core and the solvent molecules, respectively. $v_{\mathrm{b}}, v_{\mathrm{c}}$, and $v_{\mathrm{s}}$ are the respective monomeric or molecular volumes. $\mathrm{P}_{b b}, \mathrm{P}_{b c}$, and $P_{c c}$ are the partial form factors for the brush, the interference between brush and core, and the core, respectively. As can be seen from eq 19 the partial form factors are determined by the density profiles of core and brush. For an explicit evaluation of scattering data we consider for both the core and the brush rectangular shapes convoluted by Gaussians. This has the effect of rounding edges and, if the widths of the Gaussian become large, allows a continuous transition from a rectangular brush (Alexander ${ }^{5}$ and de Gennes $^{6}$ ) to a more parabolic-like profile. This would be expected according to self-consistent field calculations. ${ }^{8}$ F ourier transform of these profiles $C_{r}(z)$ yields

$$
\begin{gathered}
\mathrm{C}_{\mathrm{c}}(\mathrm{Q})=\frac{\mathrm{d} \sin \mathrm{Qd} / 2}{\mathrm{Qd} / 2} \exp \left(-\frac{1}{2} \mathrm{Q}^{2} \sigma_{\mathrm{c}}{ }^{2}\right) \\
\mathrm{C}_{\mathrm{b}}(\mathrm{Q})=\mathrm{m}_{\mathrm{s}} \frac{\mathrm{d}}{\mathrm{L}_{\mathrm{b}}}\left[2\left(\mathrm{~L}_{\mathrm{b}}+2\right) \frac{\sin \mathrm{Q}\left(\mathrm{L}_{\mathrm{b}}+\mathrm{d} / 2\right)}{\mathrm{Q}\left(\mathrm{L}_{\mathrm{b}}+\mathrm{d} / 2\right)}\right. \\
\left.\exp \left(-\frac{1}{2} \mathrm{Q}^{2} \sigma_{\mathrm{b}}{ }^{2}\right)-\mathrm{d} \frac{\sin \mathrm{Q} / 2}{\mathrm{Qd} / 2} \exp \left(-\frac{1}{2} \mathrm{Q}^{2} \sigma_{\mathrm{c}}{ }^{2}\right)\right]
\end{gathered}
$$

where $\sigma_{\mathrm{c}}$ and $\sigma_{\mathrm{b}}$ are the Gaussian widths describing the core and brush, respectively, while the expression $\mathrm{m}_{\mathrm{S}}-\rho_{\mathrm{PE}} \mathrm{M}_{\mathrm{PEP}} / 2 \sigma_{\mathrm{PEP}} \mathrm{M}_{\mathrm{PE}}$ describes the fraction of PEP in the polymer. The brush length $L_{b}$ has been defined.

The partial scattering functions $P_{r s}$ in eq 19 are $\mathrm{P}_{\mathrm{rs}}(\mathrm{Q})=\mathrm{C}_{\mathrm{r}}(\mathrm{Q}) \mathrm{C}_{\mathrm{s}}{ }^{*}(\mathrm{Q})$. Figure 4 displays $\mathrm{P}(\mathrm{Q})=\left|\mathrm{F}\left(\mathrm{Q}_{\mathrm{z}}\right)\right|^{2}$ for the different contrast situations schematically represented in Figure 5. With these form factors at hand the macroscopic cross section for a sample of isotropically oriented platel ets becomes:

$$
\frac{\mathrm{d} \Sigma}{\mathrm{d} \Omega}=\phi \frac{\phi_{\mathrm{PE}}}{\mathrm{V}_{\mathrm{PE}}} \mathrm{P}(\mathrm{Q})\left(\pi \mathrm{R}^{2}\right)^{2} \frac{\mathrm{D}(\mathrm{QR} / 2)}{\mathrm{QR} / 2}
$$

where $\phi$ is the total polymer concentration (volume fraction), $\phi_{\mathrm{P}}$ is the PE volume fraction in the diblock, and $V_{P E}=\pi R^{2} d$ is the scattering volume of the platelet core.

Structure Factor. As long as the solutions are sufficiently dilute such that the aggregates are well separated, one expects little in the way of correlations of the aggregate centers or orientations. Then eq 26 is complete since the scattering from different platelets add without interference effects. However, when the concentration is increased, correlations become more and more important yielding an extra factor $S(Q)$, the structure factor. For nonspherical particles $S(Q)$ is extremely complicated and intractable in the scope of our investigation. An exception is the stacking of large platelets driven by van der Waals interaction (even at the low concentrations observed). A treatment of this effect is given in ref 1 .

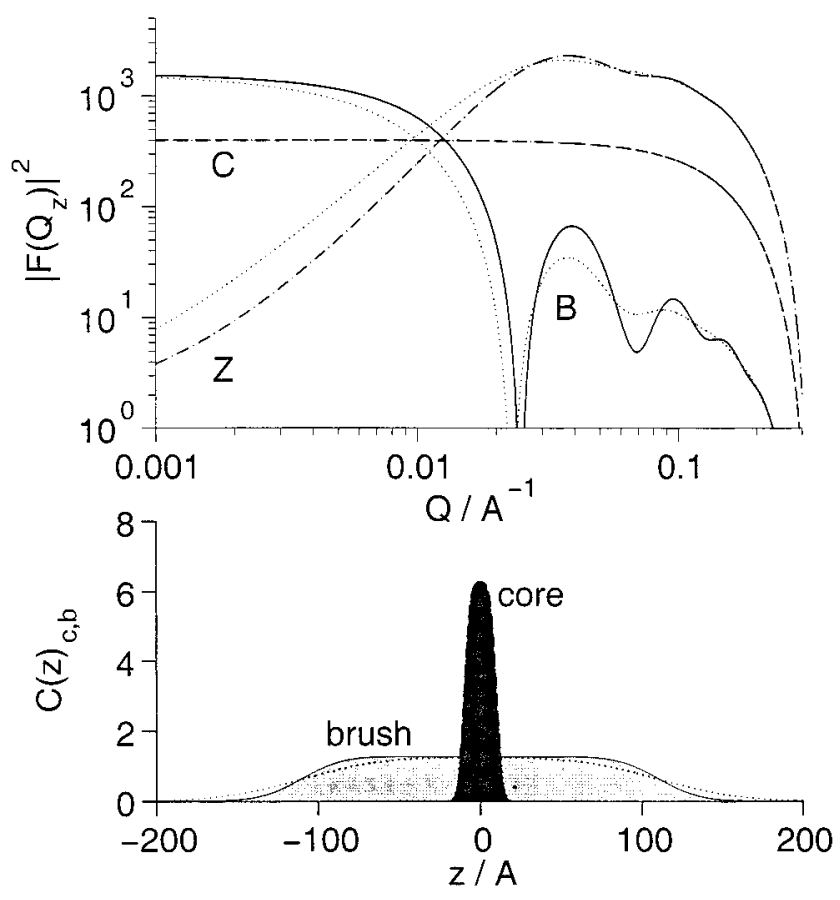

Figure 4. Form factor contribution $P(Q)=\left.F\left(Q_{z}\right)\right|^{2}$ of the plate with brushes on both sides for different contrasts. C: core contrast $\left(\Delta \xi_{\mathrm{b}}=0\right)$, B: brush contrast $\left(\Delta \xi_{\mathrm{c}}=0\right)$, Z: approximate zero average contrast $\left(\left[c_{b}(Z) \Delta \xi_{b}+c_{c}(Z) \Delta \xi_{c}\right] d_{z} \cong 0\right)$. A visualization of these contrast situations is given in Figure 5 . The lower part of this figure displays the projected average volume fractions of the brush $c_{b}(z)$ and the core $c_{c}(b)$. The dotted lines indicate the results for a larger Gaussian smearing of the brush. The factor in core contrast stays nearly constant below $\mathrm{Q}=0.1 \AA^{-1}$, whereas the brush contrast factor has a sharp minimum around $\mathrm{Q}=0.025 \AA^{-1}$ which is directly related to the brush extension. That is visible-damped by resolution effects-in the corresponding experimental curves. For exact zero average contrast the intensity drops to 0 as $\mathrm{Q} \rightarrow 0$.

Blob Scattering. The scattering intensity, see F igure 6 , considered so far, deals with the averaged density profile of the platelet. At short scales, however, the polymeric structure becomes important. Within the blobs, polymeric correlations are maintained and in addition to the above structure factor, we have to consider the scattering from the polymers in the blobs. In the simplest case of blob scattering from an ensemble of identical blobs the correlation function for one blob may be described as

$$
g(r)=r^{1 /(v-3)} \exp (-r / \xi)
$$

Fourier transformation and normalization leads to

$$
\mathrm{I}_{\text {blob }}(\mathrm{Q})=\phi \alpha \Delta \xi_{\mathrm{b}}{ }^{2} \frac{\sin [\mu \arctan (\mathrm{Q} \xi)]}{\mathrm{Q} \xi\left[1+(\mathrm{Q} \xi)^{2}\right]^{2 / 2}} \sim(\mathrm{Q} \xi)^{-1 / v}
$$

for large $\mathrm{Q}, \mathrm{v} \approx 0.6$, with $\mu=1 / \mathrm{n}-1$ and $\mathrm{a}=\mathrm{V}_{\mathrm{PEP}}$ $\mathrm{m}_{\mathrm{s}}\left(\mathrm{d} / \mathrm{L}_{\mathrm{b}}\right) \xi^{3}(1 / \mu)$

\section{Experimental Section}

Samples. The methods used for sample preparation and characterization are given in ref 1 . To utilize the full capabilities of neutron small-angle scattering (SANS) it is important to arrive at absolute intensities and to be able to control the contrasts. F or that purpose the scattering length densities of 


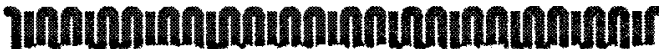

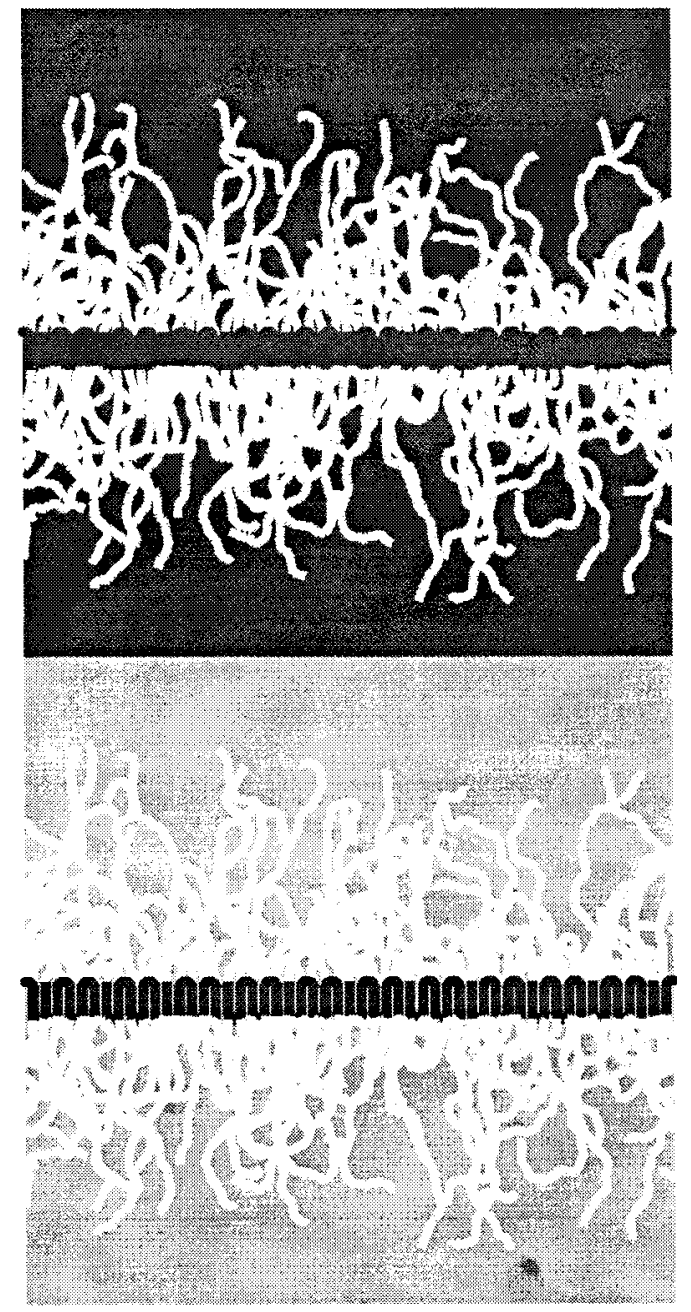

Figure 5. Idealized sketches of three different contrast situations, illustrating the real space image of the model structure. By modification of the solvent scattering length density surrounding an aggregate (e.g., dPE-hPEP) from top to bottom: core contrast, brush contrast, and zero average contrast are adjusted. It is also obvious that at shorter length scales ( $\rightarrow$ |arger $Q$ ) scattering contributions from the polymeric nature of the brush are to be expected, see text eqs 27 and 28.

the polymers involved have to be known. The hydrogenation step in the polymer synthesis introduces some uncertainties because not only is hydrogen or deuterium added to the double bonds but also the exchange of deuterium for hydrogen can occur. This phenomenon has been investigated thoroughly for the case of PE-PEP block copolymers. ${ }^{1,2}$ Furthermore, the density of the polyethylene crystals in the core is a matter of uncertainty. Again, experiments on identical but differently labeled diblock copolymers reveal ed the proper density factors. For the polymers under study, Table 1 presents characterization parameters including both the mass as well as the scattering length densities for the two lowest molecular weight samples used in this work. In addition to these two samples five other PE-PEP diblocks of varying composition and segment molecular weights were used.

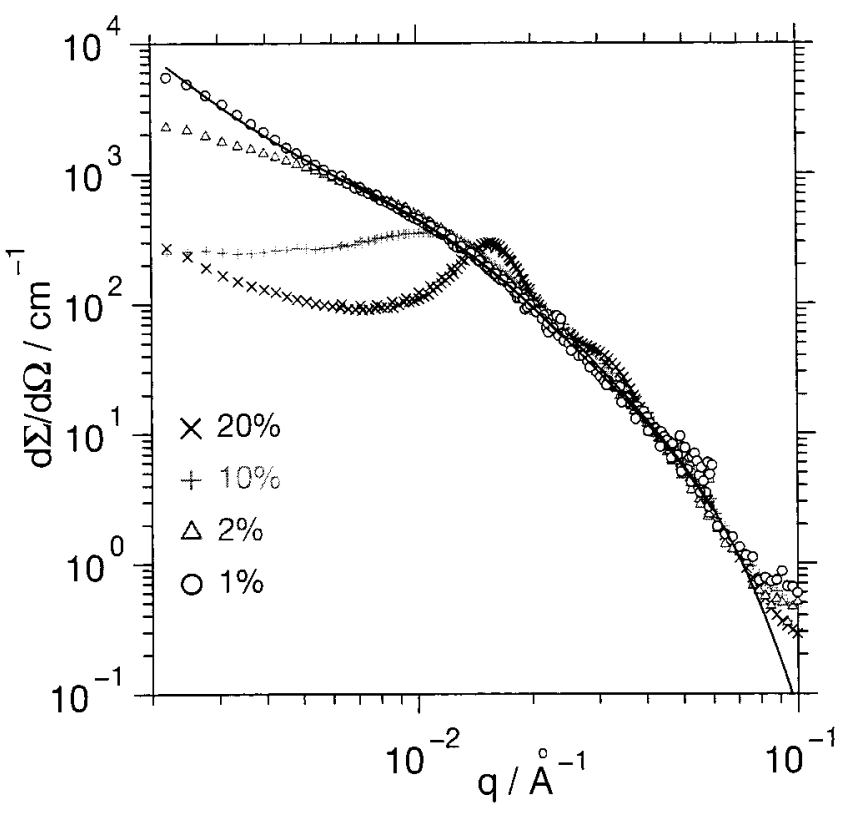

Figure 6. The scattering intensity in terms of absolute cross sections from solutions of PE/PEP diblock with $M_{w}=1.5 \mathrm{~K} / 5 \mathrm{~K}$ under core contrast at ambient temperature. The different curves correspond to different volume fractions.

Contrast variation was performed with our model solvent decane by changing the ratio of the hydrogenated and deuterated versions. The compositions of the samples are shown in Table 2. The scattering experiments were performed on polymer solutions with polymer volume fractions, $\mathrm{f}$, between 0.2 and $20 \%$. The interaction with wax was studied on solutions containing linear alkanes with 30 or 36 carbons (the alkanes $\mathrm{C}_{30}$ and $\mathrm{C}_{36}$ ) in the deuterated and protonated forms. The studies of the interactions with wax were performed in two ways:

(i) The initial series of experiments investigated the influence wax on the scattering profiles of the core or the brush. For these experiments the solvent and wax contrast was chosen so that only the core of the platelet was visible in the presence of the wax and brush (see Table 2).

(ii) In a second series the nucleation event was followed. In this case wax and core were highlighted by protonation while the solvent (see Table 2) matched the brush. All samples were prepared by addition of the required polymer and alkane fractions to the solvent with the sel ected isotope composition. Thereafter, all samples were heated to $70^{\circ} \mathrm{C}$ (a temperature above the solubilization temperature of the PE fraction and the alkanes in decane). In all cases, optically clear solutions were found. These samples were then slowly cooled to the experimental temperature (cooling rate smaller than 1deg/ min). At room temperature the solutions had a slight blue color and show a strong increase in viscosity. All samples were filled into quartz cells providing 2 or $5 \mathrm{~mm}$ thickness.

SANS. All SANS experiments were performed at the neutron small-angle diffractometer at the National Institute for Standards and Technology (NIST) in Gaithersburg, Maryland. The scattering cross sections were measured in a range of wave vectors covering $10^{-3} \leq \mathrm{Q} \leq 2 \times 10^{-1} \AA^{-1}$. The raw data were corrected for the background from the solvent, the cell, and from other sources which were measured with a blocked neutron beam. All two-dimensional scattering patterns were radially averaged and calibrated to an absolute standard in order to arrive at absolute intensities. In all fits, the resolution effects due to the incoming wavelength distribution $\Delta \lambda / \lambda \cong 0.1$, the beam collimation, and the detector pixel sizes were taken into account by proper convolution procedures. 
Table 2. SANS Experimental Parameters

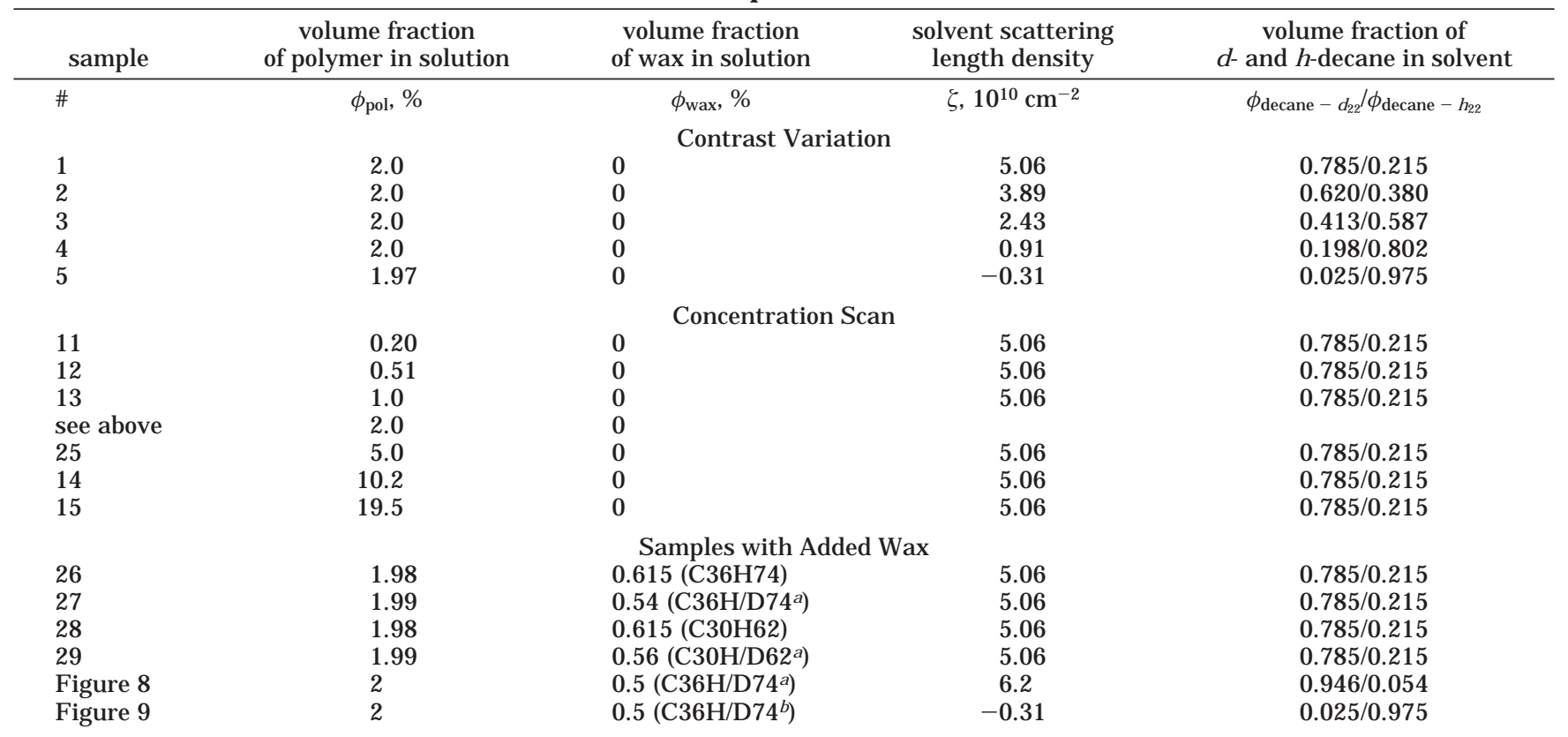

a Mixture of protonated and deuterated alkane to match scattering length densities of solvent and polymer brush. ${ }^{\mathrm{b}}$ Solvent and core scattering length densities matched.

\section{Results and Discussion}

SANS from the Low Molecular Weight Diblock; 1/1.5K. Figure 7a presents the absolute cross sections found from solutions of the 1.5/5 PE/PEP diblock under core contrast conditions. The intensity at small Q clearly indicates the formation of large aggregates. While at low volume fractions $\phi \leq 2 \%$ we observe scattering profiles without visible effects from a structure factor, i.e., aggregate-aggregate interaction. At $\phi=5 \%$ and above, a peak structure in the intermediate $\mathrm{Q}$ range evolves indicating the formation of short-range order among the platelets. Other than for the higher molecular weights investigated earlier these raw data immediately show that at low concentration no platelet aggregation occurs. Thus, the observed stapling of platelets which has been related to the van der Waals interactions between different platelets is absent. Qualitatively we may conclude that the platelets are considerably smaller than the micrometer platel ets seen for higher molecular weights.

Figure $7 \mathrm{~b}$ displays scattering data taken from a $\phi=$ $2 \%$ sol ution taken under core contrast conditions (brush is matched)-see Figure 5 top-and brush contrast conditions (core is matched), see the middle of Figure 5. The data sets display characteristic differences. While the core intensity extends to $0.08 \AA^{-1}$, the observed intensity under brush contrast drops rapidly at lower $\mathrm{Q}\left(\mathrm{Q} \approx 0.02 \AA^{-1}\right)$ indicating the much larger lateral extension of the brush compared to the core. Furthermore, above $\mathrm{Q} \cong 0.02 \AA^{-1}$ the brush data display a power law tail originating from the scattering from the blobs. This power law tail is absent under core contrast.

An attempt to fit the data with the platelet form factors developed in section 2.2 immediately showed that the separation of length scales-platel et size much larger than lateral platelet extension-is not fulfilled anymore. Therefore the situation indicated by the small Q-sphere in Figure 3 is within the observed Q-range, i.e., the actual size of the platelets shows itself within the observation range of the SANS instrument. A first attempt is to describe the scattering data in terms of an average platel et size. This effort suffers from large discrepancies in the small Q-region. Therefore in the second step we applied different distributions of platel et sizes which produced better fits than previously. The theoretical curves presented in Figure 3 display the result which is obtained using two platelets sizes namely $R_{1}=200 \AA$ and $R_{2}=2000 \AA$ at a ratio of 0.35 : 0.65 . The platelet sizes are rather ill-defined and only show the orders of magnitude. This choice of disk radii results in a satisfactory agreement between scattering data and theoretical descriptions, though most likely a somewhat broadened bimodal distributions would fit even better. Such an approach was not attempted because of the lack of uniqueness in the description.

Figure 8 displays the scattering data from the same sample now under five different solvent scattering length densities: $\xi_{s}=5.06,3.89,2.43,0.91$, and $0.31 \times$ $10^{-10} \mathrm{~cm}^{-2}$ (see Table 2). The solid lines are fitting curves with the platelet form factors taken for the same distributions as above. For all but the zero average contrast satisfactory agreement is obtained. The zero average contrast which is very sensitive (observe the long intensity scale!) to the right choice of solvent composition and the proper knowledge of the sample scattering length densities, obviously was slightly missed. The fit confirms the qualitative conclusion drawn from Figure 6, namely that no micelle stacking occurs. Furthermore, it reveals very thin core sizes of $d \cong 18 \AA$ which are accompanied by a PEP brush length of $L_{p}=$ $120 \AA$. This value can be compared with the unperturbed radius of gyration of $28 \AA$. Thus, in the selfassembled format the amorphous PEP hairs adopt a semi-stretched conformation.

We now discuss the various scaling relations, which are obtained from the considerations in section 2.2. Figure 9 presents a scaling plot for the brush length according to eq 4 . Figure 10 displays the scaling relation 

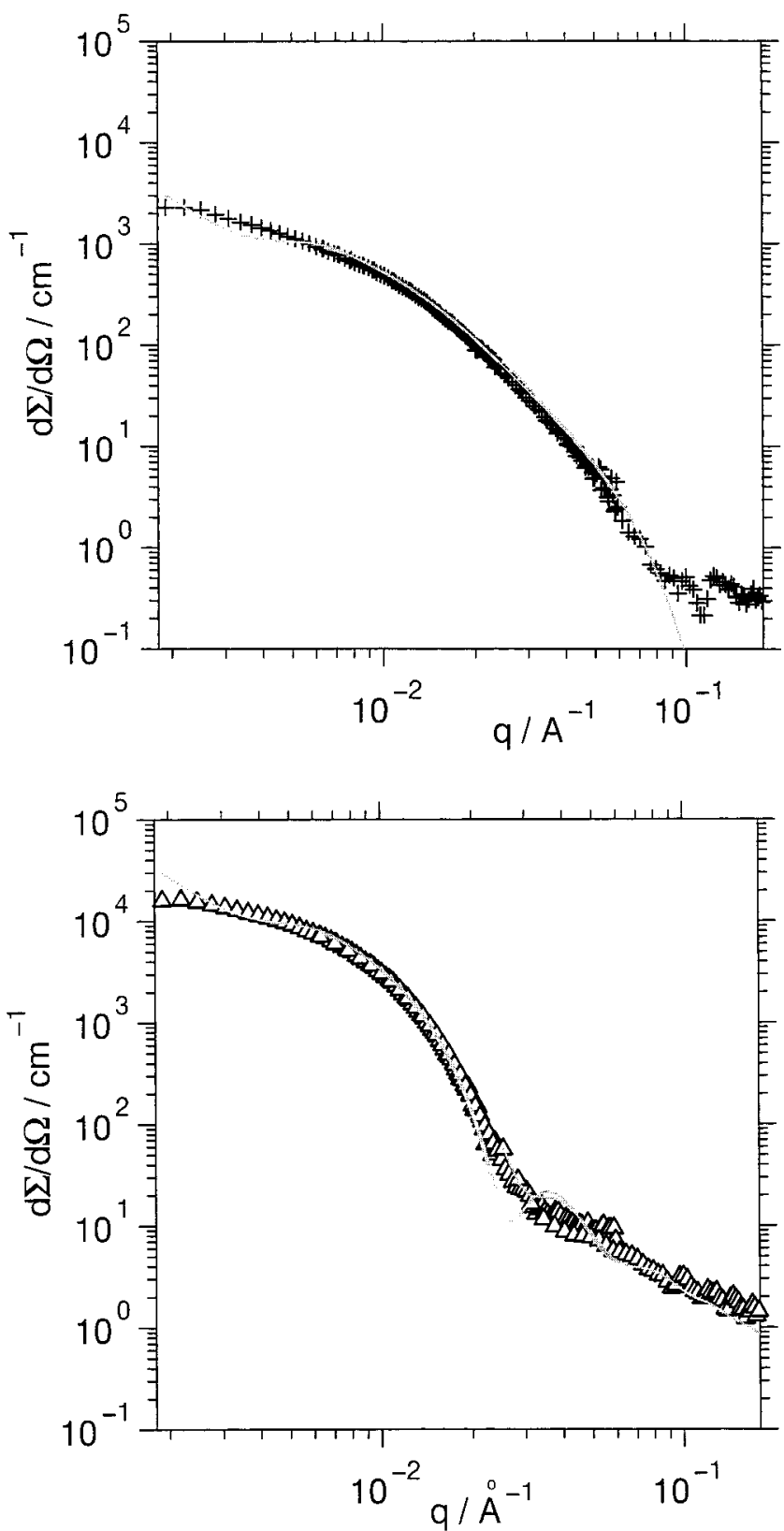

Figure 7. (a) Scattering intensity from $\phi=2 \%$ solution of $\mathrm{M}_{\mathrm{w}}=1.5 \mathrm{~K} / 5 \mathrm{~K}$ PE-PEP in decane. Core contrast as illustrated by the top part of Figure 5. (b) Scattering intensity from $\phi=$ $2 \%$ solution of $\mathrm{M}_{\mathrm{w}}=1.5 \mathrm{~K} / 5 \mathrm{~K}$ PE-PEP in decane. Lower part brush contrast as illustrated by the middle part of Figure 5 .

for the core thickness (eq 9). F rom Figure 10 we obtain the proportionality constant $C_{1}=6.7 \AA$. Inserting this result into eq 2 and inspecting then the free energy equation (eq 8) one may also cal culate the fictitious core thickness for the case where ethyl side branches are absent. Then the high fold energy $E_{f}$ in eq 8 would require a much larger core thickness represented by a constant $C_{1}=26.4 \AA$; from eq 13 . Hence, a smaller plate surface area would result. Finally, we consider the scaling relation for $L_{p}$ according to eq 10. The plot is displayed in Figure 11, and again the new data point follows the general trend of the previous result with $\mathrm{C}_{2}=3.4 \AA$.

Interaction of PE-PEP and Wax. A display of the nucleating capacity of the PE-PEP diblock $(1.5 / 5 \mathrm{~K})$ is shown in Figure 12. These micrographs were taken in

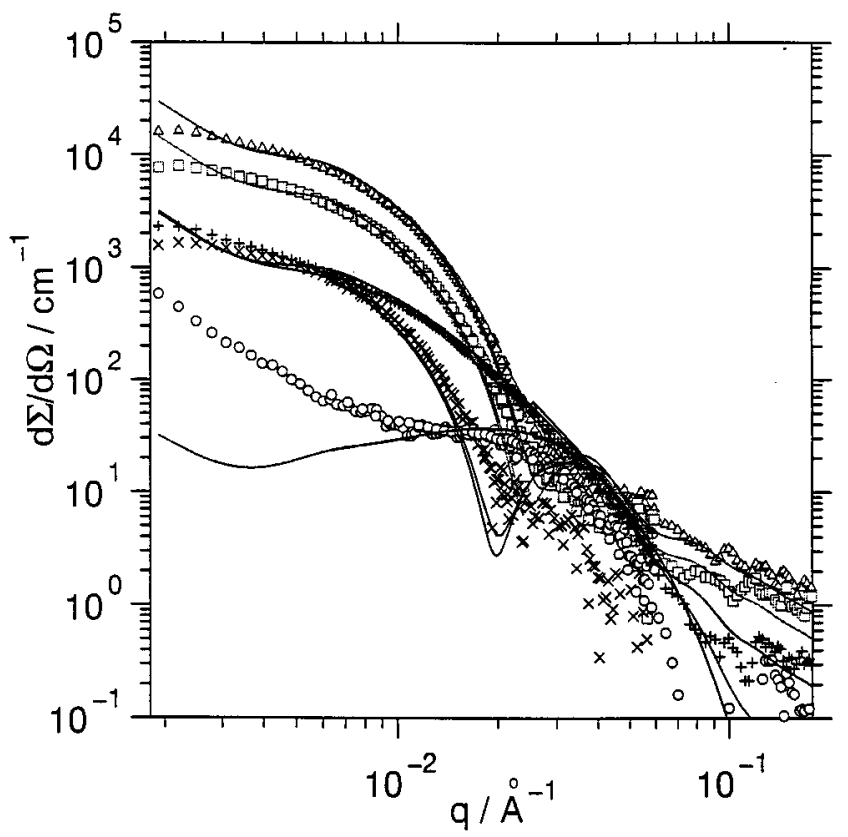

Figure 8. Data as shown in Figure 7 but for 5 different solvent contrasts (see text). The solid lines are the results of a simultaneous fit of all curves with the same structural model. The lowest curve corresponds to near zero average contrast. The large deviation-on a logarithmic scale-stems from a slight uncertainty resulting in a deviation from the exact matching condition.

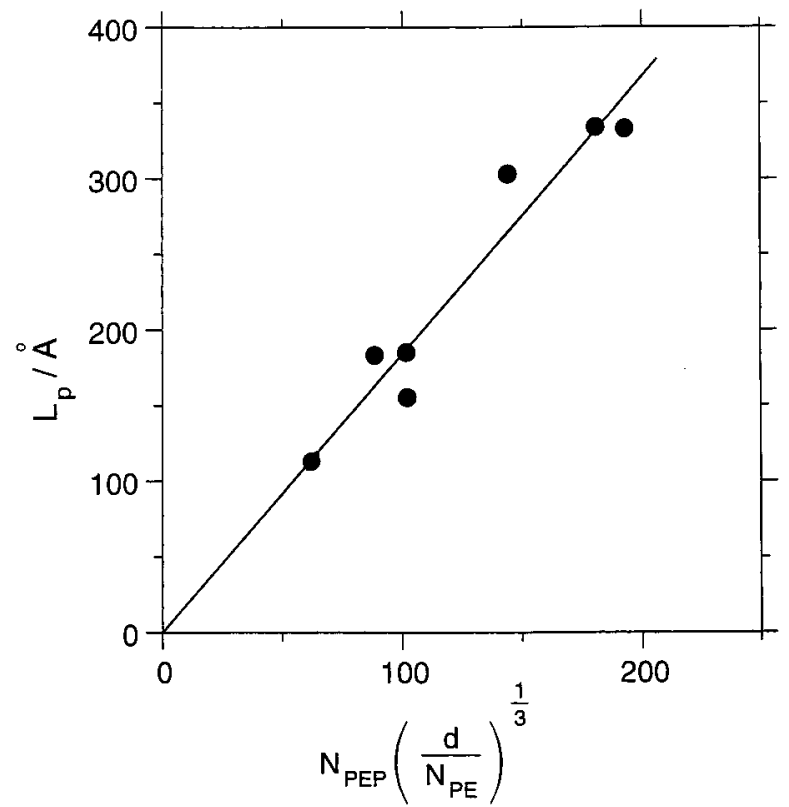

Figure 9. Scaling plot of the brush length, $L_{p}$, according to eq 4.

a cold room at $-13^{\circ} \mathrm{C}\left( \pm 1^{\circ}\right)$ and represent a commercial fuel prior to and after the addition of a formulation involving the self-assembled crystalline-amorphous diblock and a growth-arresting copolymer. The following describes our findings regarding the interplay of the PEPEP micelles with paraffins as models for wax.

In a first set of experiments we investigated the change of the platelet structure in the presence of wax. For this purpose we have prepared samples under contrast conditions where the wax and the brush or both the wax and the core were matched (see Table 2). Such matching conditions may be achieved by choosing proper 


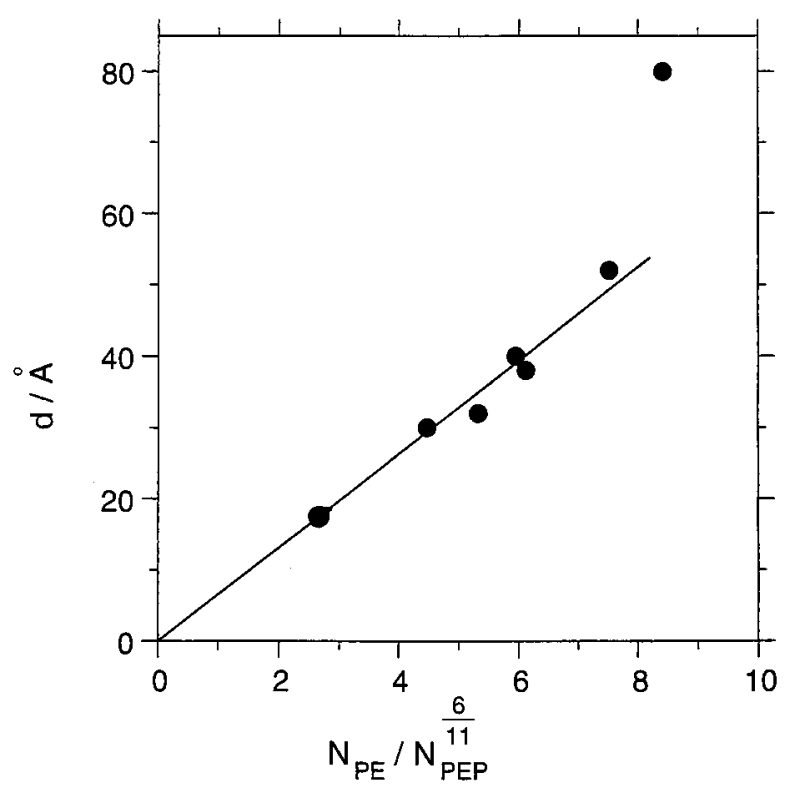

Figure 10. Scaling plot of the core thickness, d, according to eq 9.

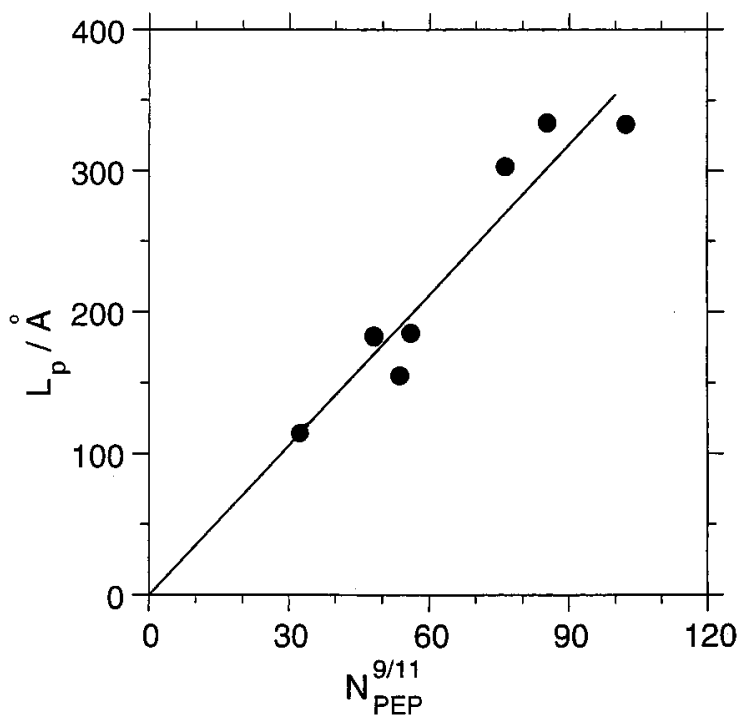

Figure 11. Scaling pl ot of the brush length, $L_{p}$, according to eq 10.

mixtures of hydrogenated and deuterated solvent and hydrogenated and deuterated wax, respectively.

Figure 13 presents SANS data from the 6/10 PE-PEP diblock copolymer under core contrast at a polymer

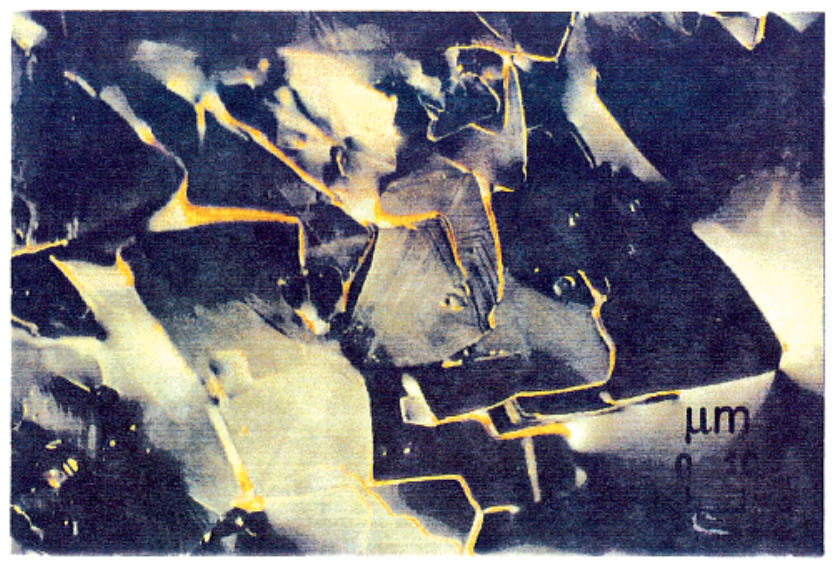

volume fraction of $\phi=2 \%$. This figure compares two scattering results: one from the pure diblock copolymer solution and the other from the same dibl ock copolymer solution including $\phi=0.5 \%$ of the paraffin $\mathrm{C}_{36}$ as a wax fraction. The contrasts in both experiments are such that only the core is visible. We realize that in the region $\mathrm{Q}>8 \times 10^{-3} \AA^{-1}$ (rel evant for the core scattering) both data sets completely agree. This experimental observation is direct proof that the wax does not influence the PE core size. Cocrystallization, which could be considered, does not occur based upon a common co-solution that was cooled from $70{ }^{\circ} \mathrm{C}$.

Figure 14 displays data from the same sample under different contrast conditions. The contrast was chosen such that only the brush is visible. Again the figure compares scattering data with and without an invisible wax fraction of $\phi=0.5 \%$. Other than in the case of the core, we realize that the brush scattering is modified. If wax is present, the dip in the intensity curves (indicating a zero in the form factor) moves to a smaller Q-value. The subsequent secondary maximum moves accordingly with $Q$ values of the order of $0.03 \AA^{-1}$ and gains intensity, indicating a much sharper brush profile.

The data have been fitted with the brush form factor according to eq 23 and 24 . The resulting profiles are displayed in the insert. With wax present the hair density profile is shifted outward compared to the empty brush indicating more stretched hairs close to the surface. This observation was taken as evidence that paraffin associates itself with the PE surface and hence forces the hair profile away from the surface.

The association of paraffins with the PE surface may be directly observed under a different contrast condition. If one chooses the contrast such that the hairs are matched but at the same time both core and paraffins have strong contrast, interaction of the wax with the PE surfaces will appear in the scattering experiment as an increase of the effective thickness of the core. F or the experiments we chose the $1.5 / 5$ sample which has a protonated core. Thus, the required contrast is achieved in taking protonated paraffins in a nearly fully deuterated solvent that matches the hairs. In this experiment we investigated the effect of two different paraffins $C_{36}$ and $\mathrm{C}_{30}$.

Figure 15 presents the scattering curves obtained at room temperature $\left(\phi_{\mathrm{PE} / \mathrm{PEP}}=2 \%, \phi_{\mathrm{wax}}=0.5\right.$ or $0.62 \%$ ). While the scattering profiles from the empty core and that from the system containing $\mathrm{C}_{30}$ coincide, a signifi-

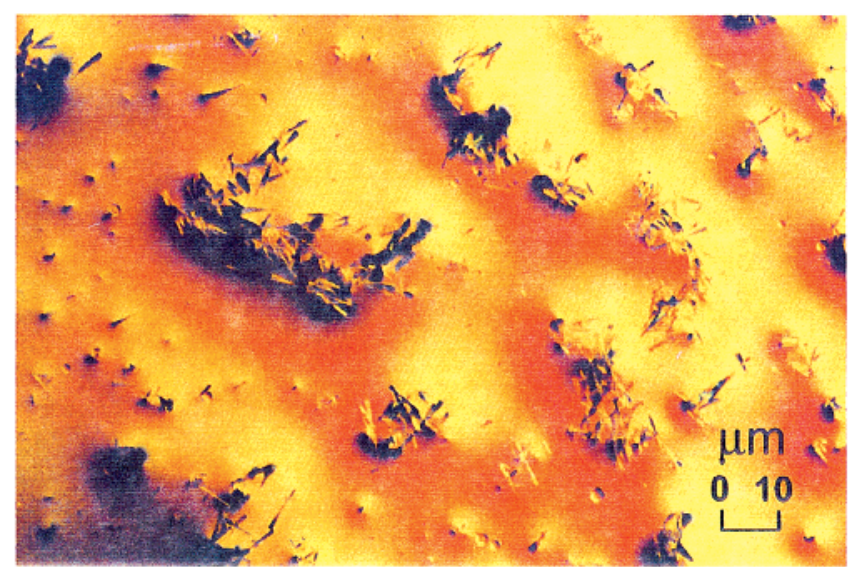

Figure 12. Micrographs, at $-13^{\circ} \mathrm{C}$, of an untreated (left side) and treated (right side) diesel fuel. 


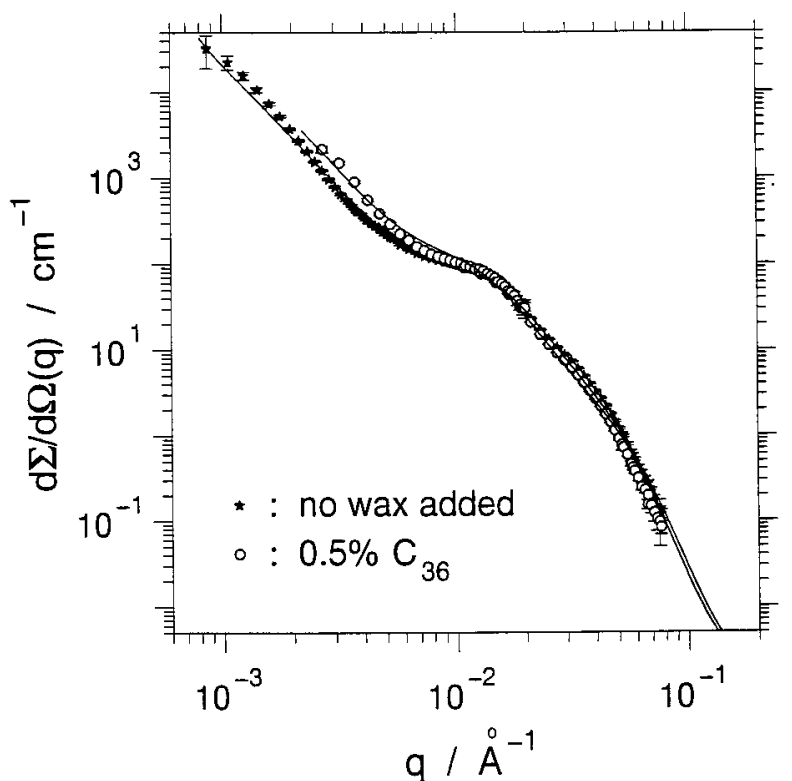

Figure 13. Scattering cross sections from the $M_{w}=6 K / 10 K$ diblock copolymer $(\phi=2 \%)$ under core contrast with and without the addition of $0.5 \% \mathrm{C}_{36}$-wax having a scattering length density equal to the solvent, i.e., invisible.

cant increase of the scattering intensity is observed for the sample containing $\mathrm{C}_{36}$. I mmediately we may con- clude that at room temperature no aggregation of $\mathrm{C}_{30}$ at the platelets occurs. On the other hand the increase of the scattering intensity for the $\mathrm{C}_{36}$ containing sample relates to wax aggregation. The solid line presents a fit with the core form factor allowing for a variation of the effective core thickness. Assuming an average surface coverage by the wax, from the fitted thickness, $d$, the aggregated wax volume fraction $\phi_{\text {wax }}$ may be obtained. Assuming identical densities for the PE and the wax crystal we have

$$
\frac{\phi_{\mathrm{pe}}+\phi_{\mathrm{wax}}}{\phi_{\mathrm{pe}}}=\frac{\mathrm{d}_{\mathrm{eff}}}{\mathrm{d}}
$$

with $\phi_{\mathrm{pe}}=0.0043, \phi_{\mathrm{C}_{36}}=0.0062, \mathrm{~d}_{\mathrm{eff}}=38 \AA$, and $\mathrm{d}=$ $18 \AA$ we arrive at $86 \%$ aggregation of the $C_{36}$ material. Finally, we note that at low Q the scattering curve for the $C_{36}$ containing sample increases more strongly than that predicted for a thicker platelet of the same size as the empty material. The increased scattering at a low Q must relate to aggregates of sizes larger than those present under wax-free conditions.

Figure 16 displays small-angle scattering results from the same samples at $\mathrm{T}=5^{\circ} \mathrm{C}$. N ow other than for the $\mathrm{T}=20^{\circ} \mathrm{C}$ result also the scattering profile obtained from the material containing $\mathrm{C}_{30}$ lies above the SANS curve

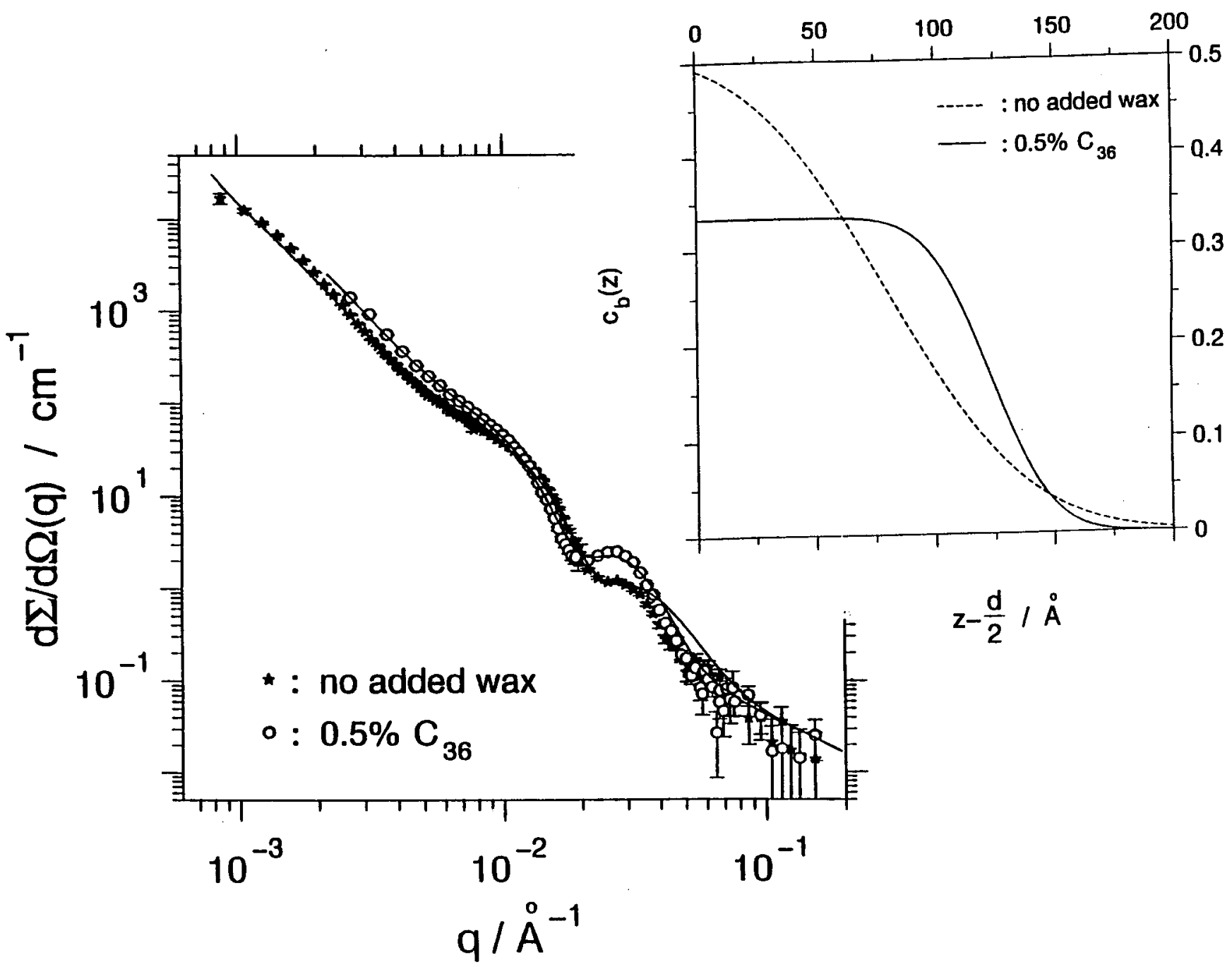

Figure 14. Scattering of the same system as shown in Figure 13 but under brush contrast. Core and wax are matched and therefore invisible. The insert shows the change of the brush concentration profile with and without wax addition. 


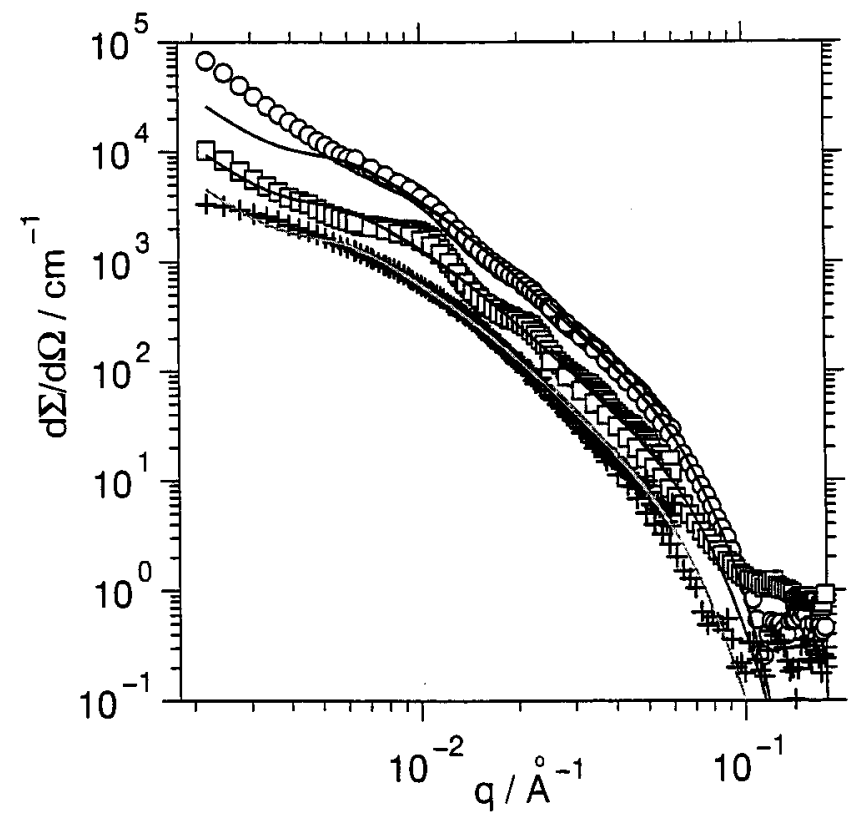

Figure 15. Scattering at ambient temperature from $M_{w}=$ $1.5 \mathrm{~K} / 5 \mathrm{~K}$ PE-PEP (2\%) under core contrast $(+)$ with $0.5 \% \mathrm{C}_{30}$ $(\square)$ and $0.62 \% \mathrm{C}_{36}(\mathrm{O})$ added. The brush is matched, core and wax are visible. Only $\mathrm{C}_{36}$ shows a strong aggregation effect.

from the empty material. Thus, both $C_{36}$ as well as $C_{30}$ aggregate at $\mathrm{T}=5^{\circ} \mathrm{C}$. We neglect the rather pronounced intermediate maxima for the $\mathrm{C}_{30}$ containing sample and fit again with the core form factors only varying the thickness. We obtain $\mathrm{d}_{\text {eff }}=25 \AA$ for the $C_{30}$ sample while

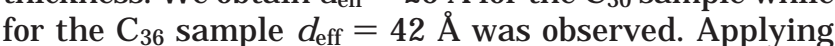
again eq 27 , we find almost $100 \%$ wax aggregation for $\mathrm{C}_{36}$ and $57 \%$ aggregation for $\mathrm{C}_{30}$.

\section{Conclusions}

We have presented a study of the self-aggregation in dilute solution and the morphology of poly(ethyleneethylene-propylene) diblock copolymers of Iow molecular weight. For the lowest molecular weight diblock we find relatively small sized platelets that do not form secondary aggregates in the form of stacks. This result agrees with earlier conjectures ${ }^{1}$ that van der Waals interactions are responsible for a stack formation needing large platelet dimensions. The observation of small platel ets and the absence of stack formation confirm the

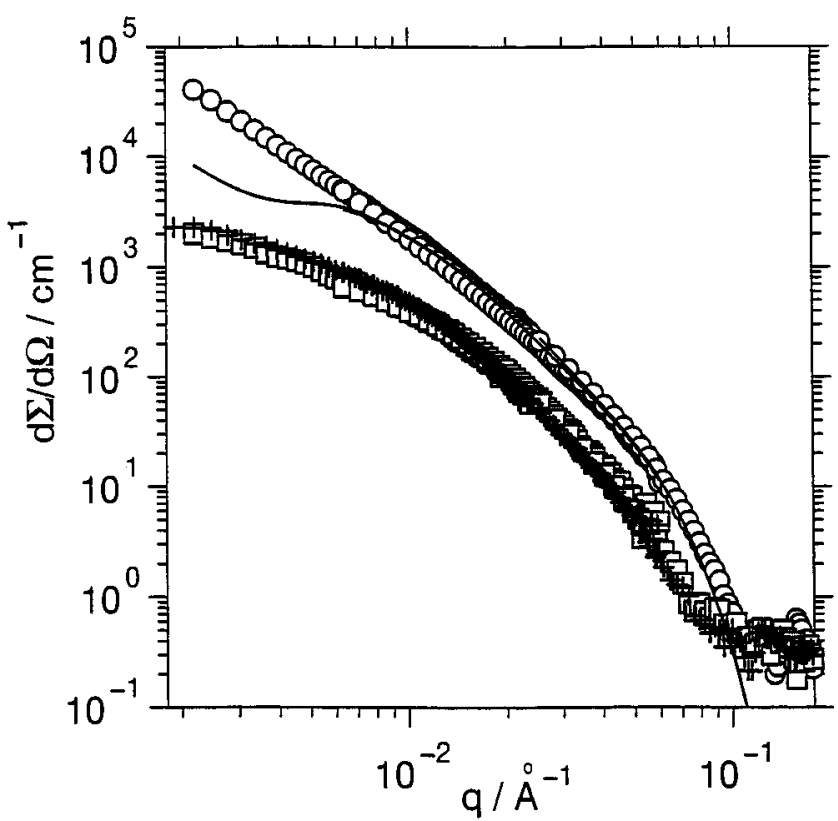

Figure 16. The same system as shown in Figure 14 but at 5 ${ }^{\circ} \mathrm{C}$. Now both waxes aggregate, see text.

earlier picture. On the basis of a free energy function for a platelet combining entropy terms from chain stretching in the brush and enthal pic terms from chain folding and defect energies in the core, scaling relations are derived which are compared with the observed lateral platelet dimensions. In all cases, earlier results based on higher molecular weight materials are confirmed extending the range of validity of these relations.

Using contrast variation the interaction of wax with the PE-PEP platelets has been investigated. While the wax does not alter the polyethylene core, we found that the brush structure is modified in the presence of waxthe density profile of the hairs is shifted outward. At a contrast where the core and the wax are seen simultaneously, the wax nucleation at the PE surface was directly observed. With decreasing temperature an increasing wax fraction is nucleated at the PE surfaces. This observation supports the suggestion that the activity of the self-assembling diblock copolymers is related to the nucleation properties of the active surface.

EF 9901544 